\title{
The power spectrum of cosmic ray arrival directions
}

\author{
M. Ahlers \\ WIPAC \& Department of Physics, University of Wisconsin-Madison, Madison, WI 53706, USA \\ Correspondence to: M. Ahlers (mahlers@icecube.wisc.edu)
}

Received: 22 June 2015 - Accepted: 9 September 2015 - Published: 7 October 2015

\begin{abstract}
Various experiments show that the arrival directions of multi- $\mathrm{TeV}$ cosmic rays show significant anisotropies at small angular scales. It was recently argued that this small scale structure may arise naturally by cosmic ray diffusion in a large-scale cosmic ray gradient in combination with deflections in local turbulent magnetic fields. We show via analytical and numerical methods that the non-trivial power spectrum in this setup is a direct consequence of Liouville's theorem and can be related to properties of relative diffusion.
\end{abstract}

\section{Introduction}

Various $\mathrm{CR}$ observatories have revealed anisotropies in the arrival directions of $\mathrm{TeV}-\mathrm{PeV}$ Galactic CRs on large and small angular scales (Amenomori et al., 2005; Amenomori, 2006; Guillian et al., 2007; Abdo et al., 2008, 2009; Bartoli et al., 2013; Aglietta et al., 2009; Abbasi et al., 2011; Aartsen et al., 2013; Abeysekara et al., 2014). The explanation of the strength and phase of the observed dipole anisotropy is challenging, but is qualitatively consistent with the diffusive prediction (Blasi and Amato, 2012; Mertsch and Funk, 2015). Medium and small scale anisotropies could be a combined effect of nearby CR sources (Salvati and Sacco, 2008; Biermann et al., 2013) and the local interstellar magnetic field structure introducing an energy-dependent magnetic mirror leakage (Drury and Aharonian, 2008), preferred CR transport directions (Malkov et al., 2010) or magnetic lenses (Battaner et al., 2011, 2015). The possible influence of the heliosphere via magnetic reconnection in the heliotail (Lazarian and Desiati, 2010), non-isotropic particle transport in the heliosheath (Desiati and Lazarian, 2013) or the heliospheric electric field structure (Drury, 2013) have also been considered. More exotic models invoke strangelet production in molecular clouds (Kotera et al., 2013) or in neutron stars (Perez-Garcia et al., 2014).

We will focus in this paper on the power spectrum of small-scale anisotropies in the arrival directions of CRs. This has been studied by IceCube (Aartsen et al., 2013) with about $1.5 \times 10^{11}$ collected CR events at median energy of $20 \mathrm{TeV}$ and more recently with HAWC (Abeysekara et al., 2014) in- cluding $4.6 \times 10^{10}$ events at a median energy of $2 \mathrm{TeV}$. The results are usually expressed in terms of the relative intensity map $\delta I$ defined by the local phase space distribution (PSD) $f$ as $\delta I \equiv(4 \pi f-n) / n$, where $n$ is the angular averaged CR density. The data points shown in Fig. 1 correspond to the multipole power spectra defined as

$$
C_{\ell}=\frac{1}{4 \pi} \int \mathrm{d} \hat{\boldsymbol{p}}_{1} \int \mathrm{d} \hat{\boldsymbol{p}}_{2} P_{\ell}\left(\hat{\boldsymbol{p}}_{1} \hat{\boldsymbol{p}}_{2}\right) \delta I\left(\hat{\boldsymbol{p}}_{1}\right) \delta I\left(\hat{\boldsymbol{p}}_{2}\right) .
$$

An average, both power spectra show a strong contribution of low- $\ell$ multipoles and a subsequent fall-off of high- $\ell$ multipole power. Note that the analysis of the power spectra in both experiments are affected by the limited field of view (FoV) and the finite size of the event samples. The limited FoV introduces cross-talk between the power of neighboring multipoles and introduces large uncertainties in the reconstruction of the low- $\ell$ multipole moments. On the other hand, the finite size of the event sample introduces noise at the level of $\mathcal{N} \simeq f_{\text {sky }} 4 \pi / N_{\mathrm{CR}}$, where $f_{\text {sky }}$ is the effective fraction of the sky that contains $N_{\mathrm{CR}}$ collected events. For IceCube we can estimate $f_{\text {sky }} \simeq 0.3$ and $\mathcal{N}_{\text {IC }} \simeq 2.5 \times 10^{-11}$ and for HAWC with $f_{\text {sky }} \simeq 0.7$ we have $\mathcal{N}_{\text {HAWC }} \simeq 1.8 \times 10^{-10}$. These different noise levels are indicated as horizontal dotted lines in Fig. 1.

It has been argued by Giacinti and Sigl (2012) that the appearance of small-scale anisotropies is a natural consequence of CR propagation in the local turbulent magnetic field with a global dipole anisotropy set by diffusion. It was subsequently shown that the behavior of the power spectra inferred from experimental data can be explained by a hierarchical transition between multipole moments in local turbulence (Ahlers, 


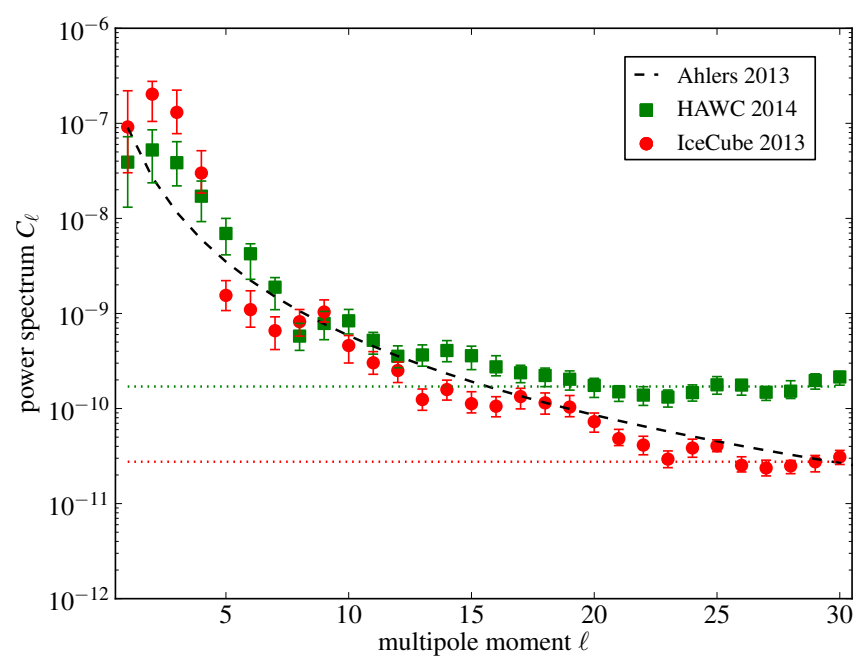

Figure 1. Observed power spectra from IceCube (Aartsen et al., 2013) and HAWC (Abeysekara et al., 2014) (from Ahlers and Mertsch, 2015). The horizontal dotted lines indicate the experimental resolution (shot noise). The dashed line indicates the asymptotic solution discussed in Ahlers (2014).

2014). The result of this analytical model is also shown as a dashed line in Fig. 1. In this paper we will summarize the results of Ahlers (2014) and the recent update of Ahlers and Mertsch (2015).

\section{Gedankenexperiment}

We first consider a case of a idealized situation where the initial PSD $\widetilde{f}$ is homogeneous but non-isotropic (Ahlers, 2014). Using Liouville's theorem, we can simply relate the PSD at a later time to the initial homogenous configuration as $f(T, \boldsymbol{r}, \boldsymbol{p})=f(0, \boldsymbol{r}(-T), \boldsymbol{p}(-T))=\widetilde{f}(\boldsymbol{p}(-T))$. Numerically, this corresponds to the backtracking of CRs with arrival orientation $\hat{p}$ along their trajectory to the initial homogeneous configuration at time $-T$. The multipole spectrum of the PSD $f(T)$ can then be simply related to the initial configuration $\widetilde{f}$ via the identity

$\frac{1}{4 \pi} \sum_{\ell \geq 0}(2 \ell+1) C_{\ell}(T)=\frac{1}{4 \pi} \int \mathrm{d} \hat{\boldsymbol{p}}_{1}\left[\tilde{f}\left(\boldsymbol{p}_{1}(-T)\right)\right]^{2}$.

This expression is for a particular magnetic field ensemble. However, for the ensemble-average we can make use of the fact that the combination of a regular and average random rotation of an isotropic distribution is eventually isotropic. More precisely, in the case of pure turbulence the average distribution is isotropic at all times, whereas for the combination of turbulent and regular magnetic fields the average distribution at intermediate times can in principle be nonisotropic but is expected to reach an isotropic state eventually. Therefore, we can relate the power spectra at different times as

$$
\frac{1}{4 \pi} \sum_{\ell \geq 0}(2 \ell+1)\left\langle C_{\ell}\right\rangle(T)=\frac{1}{4 \pi} \sum_{\ell \geq 0}(2 \ell+1) \widetilde{C}_{\ell} .
$$

Some additional assumptions have to be made to infer the power of individual multipoles. Ahlers (2014) argued that individual multipole powers decay with a rate proportional to $\ell(\ell+1)$. However, from the expression (3) we know that this process also redistributes power between multipole moments. If one makes the assumption that the transition between powers is hierarchical and the decay of $C_{\ell}$ generates only power in $C_{\ell+1}$ one can derive an asymptotic form of the relative power given by the simple expression

$\lim _{T \rightarrow \infty} \frac{\left\langle C_{\ell}\right\rangle(T)}{\left\langle C_{1}\right\rangle(T)} \simeq \frac{18}{(2 \ell+1)(\ell+2)(\ell+1)}$.

The same relative power spectrum should also be followed by the relative intensity map $\delta I$. We show the ratio (4) in Fig. (1) in comparison with the power spectrum observed with IceCube and HAWC. The scaling of $\ell>5$ multipoles is well described by the asymptotic form (Eq. 4).

Note, however, that the individual ensemble-averaged contributions $\langle C\rangle(T)$ of the model of Ahlers (2014) all decay exponentially in time. For the discussion of the expected smallscale anisotropies of a quasi-stationary diffusion state it is necessary to introduce an effective source term in form of the large-scale CR gradient. This was recently discussed by Ahlers and Mertsch (2015) and we summarize this approach in the next sections.

\section{Relative diffusion}

The previous thought experiment was designed to extract the influence of the local turbulence on the redistribution of multipole power. In order to apply these results to the realistic case of cosmic ray diffusion we have to account for source terms of the initial dipole anisotropy in the form of a cosmic ray gradient $\nabla n$. Instead of a homogeneous initial condition we can approximate this situation by assuming a quasi-stationary solution as a result of Fick's law (Jones, 1990),

$4 \pi\langle f\rangle \simeq n+(\boldsymbol{r}-3 \hat{\boldsymbol{p}} \mathbf{K}) \nabla n$,

where $\mathbf{K}$ is the diffusion tensor and $n$ the local $(\boldsymbol{r}=0) \mathrm{CR}$ density. The standard dipole prediction of the relative intensity is in this case

$$
\frac{1}{4 \pi} C_{1}=\left|\frac{\mathbf{K} \nabla n}{n}\right| .
$$

We can now use again Liouville's theorem to evaluate the sum over multipoles. For large look-back times $T$ the spatial term in the ansatz (5) dominates over the anisotropy induced 
by Fick's law. The ensemble-averaged power spectrum of the relative intensity $\delta I$ can then be evaluated as

$$
\begin{aligned}
\frac{1}{4 \pi}\left\langle C_{\ell}\right\rangle \simeq & \int \frac{\mathrm{d} \hat{\boldsymbol{p}}_{1}}{4 \pi} \int \frac{\mathrm{d} \hat{\boldsymbol{p}}_{2}}{4 \pi} P_{\ell}\left(\hat{\boldsymbol{p}}_{1} \hat{\boldsymbol{p}}_{2}\right) \\
& \times \lim _{T \rightarrow \infty}\left\langle r_{1 i}(-T) r_{2 j}(-T)\right\rangle \frac{\partial_{i} n \partial_{j} n}{n^{2}} .
\end{aligned}
$$

The sum over dipoles as in Eq. (3) is not constant in this case, but increases linearly for large backtracking times $T$. More precisely, the sum over $\ell \geq 1$ multipoles approaches asymptotically $^{1}$

$\frac{1}{4 \pi} \sum_{\ell \geq 1}(2 \ell+1)\left\langle C_{\ell}\right\rangle(T) \simeq 2 T \widetilde{K}_{i j}^{\mathrm{s}} \frac{\partial_{i} n \partial_{j} n}{n^{2}}$,

where the symmetric part of the relative diffusion tensor is defined as

$\widetilde{K}_{i j}^{\mathrm{s}}=\int \frac{\mathrm{d} \hat{\boldsymbol{p}}_{1}}{4 \pi} \int \frac{\mathrm{d} \hat{\boldsymbol{p}}_{2}}{4 \pi} \lim _{T \rightarrow \infty} \frac{1}{4 T}\left\langle\Delta r_{12 i}(-T) \Delta r_{12 j}(-T)\right\rangle$,

with $\Delta \boldsymbol{r}_{12}=\boldsymbol{r}_{1}-\boldsymbol{r}_{2}$. This shows that the effect of the generation of small-scale anisotropies from local turbulence is connected to relative diffusion. Note that the multipoles in Eq. (7) are expected to be finite in the limit of large backtracking times since particle trajectories with arbitrarily small opening angles will eventually become uncorrelated, $\left\langle r_{1 i}(-T) r_{2 j}(-T)\right\rangle \rightarrow 0$. In the next section we will describe simulations of this process that show this asymptotic behavior of the power spectrum numerically.

\section{Simulation}

We follow the approach of Giacalone and Jokipii (1999) and define a turbulent magnetic field as

$\delta \mathbf{B}(\boldsymbol{r})=\sum_{n=1}^{N} A\left(k_{n}\right)\left(\boldsymbol{a}_{n} \cos \alpha_{n}+\boldsymbol{b}_{n} \sin \alpha_{n}\right) \cos \left(\mathbf{k}_{n} \boldsymbol{r}+\beta_{n}\right)$,

where the $N$ wave vectors $k_{n}$ have random orientations in three dimensions and fixed amplitudes $k_{n}=k_{\min } e^{(n-1) \Delta \ln k}$ with $\Delta \ln k=\ln \left(k_{\max } / k_{\min }\right) / N$, i.e. equal logarithmic spacing. The parameters $\alpha_{n}$ and $\beta_{n}$ are random phases in $[0,2 \pi$ ) and the vectors $\boldsymbol{a}_{n}$ and $\boldsymbol{b}_{n}$ are defined as unit vectors in the direction $\mathbf{k}_{n} \times \mathbf{e}_{z}$ and $\mathbf{k}_{n} \times \boldsymbol{a}_{n}$, respectively. In this way the field obeys $\nabla \mathbf{B}=0$. The amplitudes $A\left(k_{n}\right)$ are defined as $A^{2}\left(k_{n}\right)=2 \sigma^{2} B_{0}^{2} G\left(k_{n}\right) / \sum_{n=1}^{N} G\left(k_{n}\right)$ with

$G\left(k_{n}\right)=4 \pi k_{n}^{2} \frac{k_{n} \Delta \ln k}{1+\left(k_{n} \lambda_{c}\right)^{\gamma}}$.

We assume that for $k_{n} \gg 1 / \lambda_{\mathrm{c}}$ the turbulence follows a Kolmogorov-type turbulence with $\gamma=11 / 3$. The scaling factor $\sigma^{2}$ is normalized such that $\sigma^{2}=U_{\delta B} / U_{B_{0}}$ and $\lambda_{\mathrm{c}}$ is a wave vector corresponding to the coherence scale of the turbulent magnetic field.

\footnotetext{
${ }^{1}$ We use the identity $\delta(x-y)=\sum_{\ell} \frac{2 \ell+1}{2} P_{\ell}(x) P_{\ell}(y)$ and $P_{\ell}(1)=1$.
}

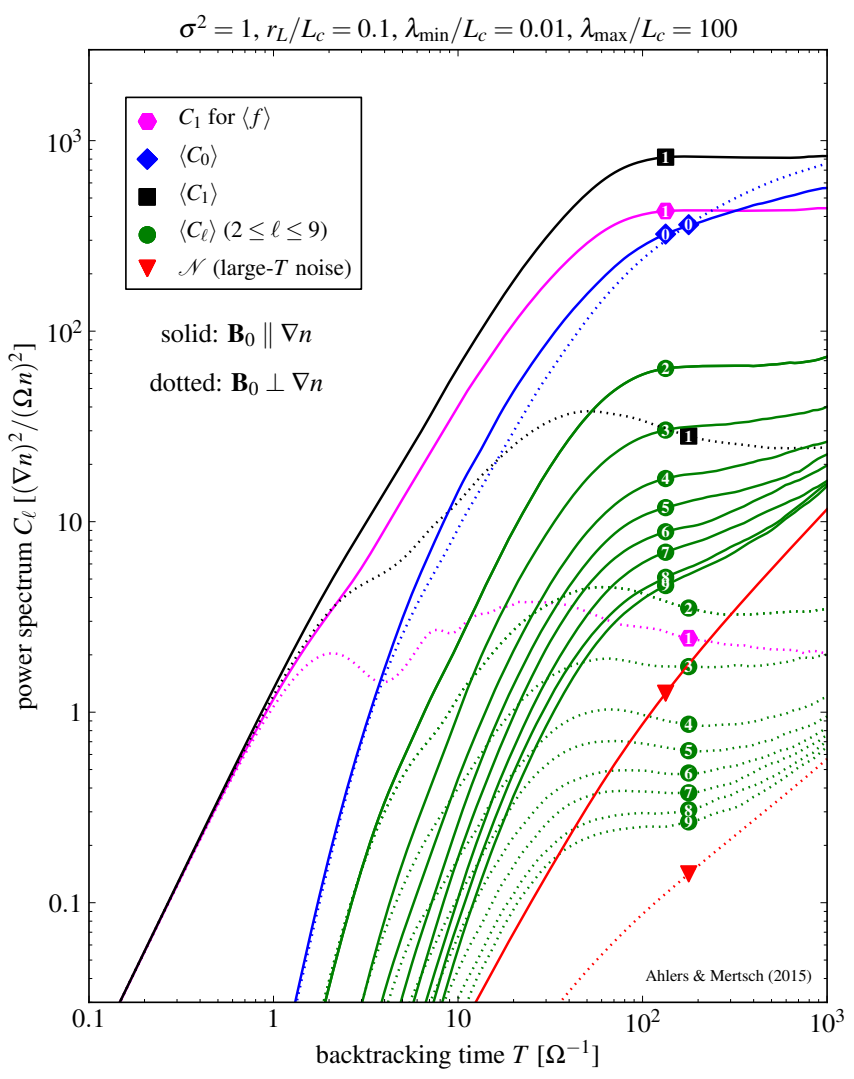

Figure 2. The evolution of the ensemble-averaged power spectrum (Eq. 7) in units of $\Omega=e B_{0} / p_{0}$ for a CR gradient parallel (solid lines) and perpendicular (dotted lines) to the regular magnetic field and the 3-D turbulence model discussed in the main text (see also Ahlers and Mertsch, 2015). We show results in terms of the dipole $\left\langle C_{1}\right\rangle$ (black), monopole $\left\langle C_{0}\right\rangle$ (blue) and medium$\ell$ multipoles (green). We also show the asymptotic noise level (Eq. 12) (red) and the dipole prediction (Eq. 6) of standard diffusion (magenta) evaluated by the replacement $\left\langle r_{1 i} r_{2 j}\right\rangle \rightarrow\left\langle r_{1 i}\right\rangle\left\langle r_{2 j}\right\rangle$ in Eq. (7).

In our simulation we assume $\sigma^{2}=1$ and choose $\lambda_{\min }=$ $0.01 \lambda_{\mathrm{c}}$ and $\lambda_{\max }=100 \lambda_{\mathrm{c}}$ with $k=2 \pi / \lambda$. We fix the rigidity of the CRs to $r_{\mathrm{L}}=0.1 \lambda_{\mathrm{C}}$. We sample over $N_{B}=10$ different turbulent magnetic field configurations. For each field configuration we uniformly sample $N_{\text {pix }}=12288 \mathrm{CR}$ orientation $\hat{\boldsymbol{p}}_{i}(0)$ following the HEALPix parametrization $\left(n_{\text {side }}=32\right)$ (Gorski et al., 2005), that we track back through the static magnetic field $\mathbf{B}(\mathbf{r})=B_{0} \hat{\mathbf{e}}_{z}+\delta \mathbf{B}(\mathbf{r})$ to find the initial orientation $\hat{\boldsymbol{p}}_{i}(-T)$ and position $\mathbf{r}_{i}(-T)$. Figure 2 shows the power spectrum at different times determined via a multipole expansion of the maps using the HEALPix utilities.

At large times the high- $\ell$ components in the multipole expansion are dominated by shot noise, due to the finite sample size and the uncorrelated random walk at large back-tracking 
times. This noise level can be estimated as

$\mathcal{N} \simeq \frac{4 \pi}{N_{\text {pix }}} 2 T K_{i j} \frac{\partial_{i} n \partial_{j} n}{n^{2}}$.

Therefore, for large backtracking times $T$ the map is eventually dominated by noise. However, for sufficiently low multipole moments and large sample size $N_{\text {pix }}$ the stable asymptotic behavior of low- $\ell$ multipoles can be recovered.

\section{Conclusions}

We have discussed in this paper the generation of small-scale anisotropy from CR propagation in local magnetic turbulence. We have shown that with minimal assumptions about the decay of and transition between multipole powers one can derive an asymptotic solution that is consistent with observations of IceCube and HAWC. We have discussed our recent approach to bring these results into a more realistic setup of $\mathrm{CR}$ diffusion with a large-scale $\mathrm{CR}$ gradient. One can show analytically that the generation of multipole power is related to properties of relative CR diffusion. This was supported by numerical simulations.

Acknowledgements. The author would like to thank Philipp Mertsch for collaboration and the organizers of Cosmic Ray Anisotropies 2015 for a very pleasant conference. This work is supported by the National Science Foundation under grants OPP-0236449 and PHY-0236449.

Edited by: P. Desiati

Reviewed by: two anonymous referees

\section{References}

Aartsen, M. G., Abbasi, R., Abdou, Y., et al. (IceCube Collaboration): The IceCube Neutrino Observatory Part III: Cosmic Rays, arXiv:1309.7006, 2013.

Abbasi, R., Abdou, Y., Abu-Zayyad, T., et al. (IceCube Collaboration): Observation of Anisotropy in the Arrival Directions of Galactic Cosmic Rays at Multiple Angular Scales with IceCube, Astrophys. J., 740, 16, doi:10.1088/0004-637X/740/1/16, 2011.

Abdo, A. A., Allen, B., Aune, T., et al.: Discovery of Localized Regions of Excess 10-TeV Cosmic Rays, Phys. Rev. Lett., 101, 221101, doi:10.1103/PhysRevLett.101.221101, 2008.

Abdo, A. A., Allen, B., Aune, T., et al.: The Large Scale CosmicRay Anisotropy as Observed with Milagro, Astrophys. J., 698, 2121-2130, 2009.

Abeysekara, A., Alfaro, R., Alvarez, C., et al. (The HAWC Collaboration): Observation of Small-scale Anisotropy in the Arrival Direction Distribution of TeV Cosmic Rays with HAWC, 796, 108, doi:10.1088/0004-637X/796/2/108, 2014.

Aglietta, M., Alekseenko, V. V., Alessandro, B., Antonioli, P., Arneodo, F., Bergamasco, L., Bertaina, M., Bonino, R., Castellina, A., and Chiavassa, A.: Evolution of the cosmic ray anisotropy above $10^{14} \mathrm{eV}$, Astrophys. J., 692, L130-L133, 2009.
Ahlers, M.: Anomalous Anisotropies of Cosmic Rays from Turbulent Magnetic Fields, Phys. Rev. Lett., 112, 021101, doi:10.1103/PhysRevLett.112.021101, 2014.

Ahlers, M. and Mertsch, P.: Small-Scale Anisotropies of Cosmic Rays from Relative Diffusion, arXiv:1506.05488, 2015.

Amenomori, M.: Anisotropy and Corotation of Galactic Cosmic Rays, Science, 314, 439-443, 2006.

Amenomori, M., Ayabe, S., Cui, S. W., et al. (The Tibet AS? Collaboration): Large-scale sidereal anisotropy of Galactic cosmic-ray intensity observed by the Tibet air shower array, Astrophys. J., 626, L29-L32, doi:10.1086/431582, 2005.

Bartoli, B., Bernardini, P., Bi, X. J., et al. (ARGO-YBJ Collaboration): Medium scale anisotropy in the $\mathrm{TeV}$ cosmic ray flux observed by ARGO-YBJ, Phys. Rev. D, 88, 082001, doi:10.1103/PhysRevD.88.082001, 2013.

Battaner, E., Castellano, J., and Masip, M.: Cosmic magnetic lenses, Astron. Astrophys., 527, A79, doi:10.1051/00046361/201015663, 2011.

Battaner, E., Castellano, J., and Masip, M.: Magnetic fields and cosmic ray anisotropies at $\mathrm{TeV}$ energies, Astrophys. J., 799, 157, doi:10.1088/0004-637X/799/2/157, 2015.

Biermann, P. L., Becker, J., Seo, E.-S., and Mandelartz, M.: Cosmic ray transport and anisotropies, Astrophys.J., 768, 124, doi:10.1088/0004-637X/768/2/124, 2013.

Blasi, P. and Amato, E.: Diffusive propagation of cosmic rays from supernova remnants in the Galaxy. II: anisotropy, J. Cosmol. Astropart. P., 1201, 011, doi:10.1088/1475-7516/2012/01/011, 2012.

Desiati, P. and Lazarian, A.: Anisotropy of TeV Cosmic Rays and the Outer Heliospheric Boundaries, Astrophys. J., 762, 44, doi:10.1088/0004-637X/762/1/44, 2013.

Drury, L. O'C.: The problem of small angular scale structure in the cosmic ray anisotropy data, 33rd International Cosmic Ray Conference (ICRC2013), 2-9 July 2013, Rio de Janeiro, Brazil, 2013.

Drury, L. O'C. and Aharonian, F.: The puzzling MILAGRO hot spots, Astropart. Phys., 29, 420-423, 2008.

Giacalone, J. and Jokipii, J.: The Transport of Cosmic Rays across a Turbulent Magnetic Field, Astrophys. J., 520, 204-214, 1999.

Giacinti, G. and Sigl, G.: Local Magnetic Turbulence and TeVPeV Cosmic Ray Anisotropies, Phys. Rev. Lett., 109, 071101, doi:10.1103/PhysRevLett.109.071101, 2012.

Gorski, K., Hivon, E., Banday, A., Wandelt, B., Hansen, F., Reinecke, M., and Bartelmann, M.: HEALPix - A Framework for high resolution discretization, and fast analysis of data distributed on the sphere, Astrophys. J., 622, 759-771, 2005.

Guillian, G., Hosaka, J., Ishihara, K., et al. (Super-Kamiokande Collaboration): Observation of the anisotropy of $10-\mathrm{TeV}$ primary cosmic ray nuclei flux with the super-kamiokande-I detector, Phys. Rev. D, 75, 062003, doi:10.1103/PhysRevD.75.062003, 2007.

Jones, F. C.: The generalized diffusion-convection equation, Astrophys. J., 361, 162-172, 1990.

Kotera, K., Perez-Garcia, M. A., and Silk, J.: Strangelets and the TeV-PeV cosmic-ray anisotropies, Phys. Lett. B, 725, 196-199, 2013.

Lazarian, A. and Desiati, P.: Magnetic reconnection as the cause of cosmic ray excess from the heliospheric tail, Astrophys. J., 722, 188-196, 2010. 
Malkov, M., Diamond, P., Drury, L., and Sagdeev, R.: Probing Nearby CR Accelerators and ISM Turbulence with Milagro Hot Spots, Astrophys. J., 721, 750-761, 2010.

Mertsch, P. and Funk, S.: Solution to the cosmic ray anisotropy problem, Phys. Rev. Lett., 114, 021101, doi:10.1103/PhysRevLett.108.211102, 2015.
Perez-Garcia, M. A., Kotera, K., and Silk, J.: Anisotropy in Cosmic rays from internal transitions in neutron stars, Nucl. Instrum. Methods A, 742, 237-240, 2014.

Salvati, M. and Sacco, B.: The Milagro anti-Center hot spots: cosmic rays from the Geminga Supernova?, Astron. Astrophys., 485, 527-529, 2008. 